\title{
Mathematical Properties of the Elasticity Difference Tensor
}

\author{
E.G.L.R. Vaz* and Irene Brito* \\ * Departamento de Matemática para a Ciência e Tecnologia, Universidade do Minho, Campus de \\ Azurém,4800-058 Guimarães, Portugal - evaz@mct.uminho.pt
}

\begin{abstract}
A tetrad, adapted to the principal directions of the unstrained reference tensor, is chosen and the elasticity difference tensor, as introduced in [1], is decomposed along those directions. The second order tensors obtained are studied and an example is presented.
\end{abstract}

Keywords: elasticity; classification

PACS: 04.20.-q

\section{INTRODUCTION}

Here we will consider a continuous medium possessing elastic properties, the collection of all its idealized particles being the 3 -dimensional space $X$ - the material space. $(M, g)$ represents the space-time manifold, i.e. $M$ is a four-dimensional, connected, Hausdorff manifold and $g$ is a Lorentzian metric with signature $(-+++)$ such that $g=-\mathbf{u} \otimes \mathbf{u}+h$, where: (i) $h=\pi^{*} k, \pi^{*}$ being the usual canonical projection onto $X$ and $k$ being a metric in $X$; (ii) $\pi^{-1}(p \in X)$ defines a timelike curve in $M$ having $\mathbf{u}$ as unit tangent vector field and represents the flowline of $p$; (iii) $\pi: U \subset M \longrightarrow X$ describes a state of matter.

Following [1], for an unrelaxed state of matter the unstrained reference tensor [2] can be written as $k_{a b}=n_{1}^{2} x_{a} x_{b}+n_{2}^{2} y_{a} y_{b}+n_{3}^{2} z_{a} z_{b}$, the scalar fields $n_{1}, n_{2}, n_{3}$ being related to the eigenvalues along the principal directions of $k_{b}^{a}$. An orthonormal tetrad $\{\mathbf{u}, \mathbf{x}, \mathbf{y}, \mathbf{z}\}$, with $\mathbf{u}$ timelike and $\mathbf{x}, \mathbf{y}, \mathbf{z}$ unit spacelike vector fields aligned with the eigenvectors of $k_{a}^{b}$, will be used. On a local coordinate system, the metric $g$ can be written as

$$
g_{a b}=-u_{a} u_{b}+x_{a} x_{b}+y_{a} y_{b}+z_{a} z_{b} .
$$

In order to study elasticity properties of the space-time, the authors in [1] define the elasticity difference tensor:

$$
S_{b c}^{a}=\frac{1}{2} k^{a m}\left(D_{b} k_{m c}+D_{c} k_{m b}-D_{m} k_{b c}\right),
$$

where $D$ denotes projected covariant derivative associated to $g$. A classification of $S$ will certainly be interesting for the characterization of the elasticity properties of the space-time. In order to do so, we decompose $S_{b c}^{a}$ along the principal directions of $k_{b}^{a}$ :

$$
S_{b c}^{a}=M_{b c} x^{a}+N_{b c} y^{a}+P_{b c} z^{a} .
$$

The second order symmetric tensors $M, N, P$ are now investigated. 


\section{MAIN RESULTS. AN EXAMPLE}

The following results for $M_{b c}$ were obtained, the proofs being in [4].

Theorem 1 The general form of $M_{b c}$ is given by

$$
\begin{aligned}
& M_{b c}=u^{m}\left(x_{m ;(b} u_{c)}+u_{(b} x_{c) ; m}\right)+x_{(b ; c)}-x^{m} x_{(c} x_{b) ; m}+\gamma_{011} u_{(b} x_{c)}-\gamma_{010} u_{b} u_{c} \\
& +\frac{1}{n_{1}}\left[2 n_{1,(b} x_{c)}+2 n_{1, m} u^{m} u_{(b} x_{c)}+n_{1, m} x^{m} x_{b} x_{c}\right] \\
& +\frac{1}{n_{1}^{2}}\left\{-x^{m}\left(z_{b} z_{c} n_{3} n_{3, m}+y_{b} y_{c} n_{2} n_{2, m}\right)+n_{2}^{2}\left[\left(\gamma_{021}-\gamma_{120}\right) u_{(b} y_{c)}+x^{m}\left(y_{m ;(b} y_{c)}-y_{(b} y_{c) ; m}\right)\right]\right. \\
& \left.+n_{3}^{2}\left[\left(\gamma_{031}-\gamma_{130}\right) u_{(b} z_{c)}+x^{m}\left(z_{m ;(b} z_{c)}-z_{(b} z_{c) ; m}\right)\right]\right\}
\end{aligned}
$$

where $\gamma_{a b c}$ are the rotation coefficients and a comma represents a partial derivative.

Theorem $2 \boldsymbol{x}$ is an eigenvector of $M_{b c}$ iff $n_{1}$ remains invariant along the directions of $\boldsymbol{y}$ and $z$, i.e. $\Delta_{y}\left(\log n_{1}\right)=\Delta_{z}\left(\log n_{1}\right)=0$, where $\Delta_{y}$ represents the intrinsic derivative along y. The corresponding eigenvalue is $\lambda=\Delta_{x}\left(\log n_{1}\right)$.

Theorem $3 \boldsymbol{y}$ is an eigenvector of $M_{b c}$ iff $n_{1}$ remains invariant along the direction of $\boldsymbol{y}$, i.e. $\Delta_{y}\left(\log n_{1}\right)=0$, and $\frac{1}{2} \gamma_{132}\left[-\left(n_{3}^{2} / n_{1}^{2}\right)+1\right]+\frac{1}{2} \gamma_{123}\left[1-\left(n_{2}^{2} / n_{1}^{2}\right)\right]+\frac{1}{2} \gamma_{231}\left[\left(n_{3}^{2} / n_{1}^{2}\right)-\right.$ $\left.\left(n_{2}^{2} / n_{1}^{2}\right)\right]=0$. The corresponding eigenvalue is $\lambda=-\left(n_{2} / n_{1}^{2}\right) \Delta_{x} n_{2}+\gamma_{122}\left[-\left(n_{2}^{2} / n_{1}^{2}\right)+1\right]$.

Theorem $4 z$ is an eigenvector of $M_{b c}$ iff $n_{1}$ remains invariant along the direction of $z$, i.e. $\Delta_{z}\left(\log n_{1}\right)=0$, and $\frac{1}{2} \gamma_{123}\left[1-\left(n_{2}^{2} / n_{1}^{2}\right)\right]+\frac{1}{2} \gamma_{132}\left[1-\left(n_{3}^{2} / n_{1}^{2}\right)\right]+\frac{1}{2} \gamma_{231}\left[\left(n_{3}^{2} / n_{1}^{2}\right)-\right.$ $\left.\left(n_{2}^{2} / n_{1}^{2}\right)\right]=0$. The corresponding eigenvalue is $\lambda=-\left(n_{3} / n_{1}^{2}\right) \Delta_{x} n_{3}-\gamma_{133}\left[\left(n_{3}^{2} / n_{1}^{2}\right)-1\right]$.

Similar results have been obtained by the authors for $N$ and $P$ [4].

The following example illustrates the results above. We consider a spherically symmetric metric $g$ written in local coordinates $t, r, \theta, \phi$ as $d s^{2}=-d t^{2}+d r^{2}+r^{2} d \theta^{2}+$ $r^{2} \sin ^{2} \theta d \phi^{2}$ (see [3], p.186). If a radial deformation is considered such that $d s^{2}=$ $-d t^{2}+n^{2}(r)\left[d r^{2}+r^{2} d \theta^{2}+r^{2} \sin ^{2} \theta d \phi^{2}\right]$, the only non-zero components of the elasticity difference tensor are $S_{r r}^{r}=S_{\theta r}^{\theta}=S_{\phi r}^{\phi}=\frac{1}{n(r)} \frac{d n(r)}{d r}$ and $S_{\phi \phi}^{r}=-\frac{r^{2} \sin ^{2}(\theta)}{n(r)} \frac{d n(r)}{d r}=\sin ^{2}(\theta) S_{\theta \theta}^{r}$. Then $M_{b c}=\lambda_{1}\left(x_{b} x_{c}-y_{b} y_{c}-z_{b} z_{c}\right), N_{b c}=2 \lambda_{2}\left(x_{b} y_{c}+x_{c} y_{b}\right)$ and $P_{b c}=2 \lambda_{3}\left(x_{b} z_{c}+x_{c} z_{b}\right)$, where $\lambda_{1}=\lambda_{2}=\lambda_{3}=\frac{1}{n(r)} \frac{d n(r)}{d r}$. Therefore, the eigenvalue associated with the eigenvector $\mathbf{u}$ vanishes identically. The remaining eigenvectors are: (i) $\{\mathbf{x}, \mathbf{y}, \mathbf{z}\}$ for $M_{b c}, \frac{1}{n(r)} \frac{d n(r)}{d r}$, $-\frac{1}{n(r)} \frac{d n(r)}{d r},-\frac{1}{n(r)} \frac{d n(r)}{d r}$ being the corresponding eigenvalues, so that the Segre type is $\{1,1(11)\}$; (ii) $\{\mathbf{x}+\mathbf{y}, \mathbf{x}-\mathbf{y}, \mathbf{z}\}$ for $N_{b c}$ with eigenvalues $\frac{1}{n(r)} \frac{d n(r)}{d r},-\frac{1}{n(r)} \frac{d n(r)}{d r}$ and zero, respectively, the Segre type being $\{1,111\}$; (iii) $\{\mathbf{x}+\mathbf{z}, \mathbf{x}-\mathbf{z}, \mathbf{y}\}$ for $P_{b c}$ with eigenvalues $\frac{1}{n(r)} \frac{d n(r)}{d r},-\frac{1}{n(r)} \frac{d n(r)}{d r}$ and zero, respectively, the Segre type being then $\{1,111\}$.

\section{REFERENCES}

1. M. Karlovini, and L. Samuelsson, Class.Quantum Grav., 20 (2003).

2. B. Carter and H. Quintana, Proc. R. Soc., A 331, 57 (1972).

3. R. Inverno, Introducing Einstein's Relativity, Clarendon Press, Oxford (1996).

4. E.G.L.R. Vaz and Irene Brito, preprint. 